\title{
DE FINETTI THEOREM ON THE CAR ALGEBRA
}

\author{
VITONOFRIO CRISMALE AND FRANCESCO FIDALEO
}

\begin{abstract}
The symmetric states on a quasi local $C^{*}$-algebra on the infinite set of indices $J$ are those invariant under the action of the group of the permutations moving only a finite, but arbitrary, number of elements of $J$. The celebrated De Finetti Theorem describes the structure of the symmetric states (i.e. exchangeable probability measures) in classical probability. In the present paper we extend De Finetti Theorem to the case of the CAR algebra, that is for physical systems describing Fermions. Namely, after showing that a symmetric state is automatically even under the natural action of the parity automorphism, we prove that the compact convex set of such states is a Choquet simplex, whose extremal (i.e. ergodic w.r.t. the action of the group of permutations previously described) are precisely the product states in the sense of Araki-Moriya. In order to do that, we also prove some ergodic properties naturally enjoyed by the symmetric states which have a self-containing interest.
\end{abstract}

Mathematics Subject Classification: 46L53, 46L05, 60G09, 46L30, 46N50.

Key words: Non commutative probability and statistics; $C^{*_{-}}$ algebras, states; Exchangeability; Applications to quantum physics.

\section{INTRODUCTION}

Exchangeable or, equivalently, symmetrically dependent sequences of random variables and symmetric states have been investigated in a wide way both in Probability Theory and Operator Algebras.

After De Finetti's pioneering work [13] for 2-point valued random variables, it has been shown that more and more general sequences of exchangeable random variables are mixtures of independent identical distributed (i.i.d. for short) sequences. One of its most general version in classical probability was obtained by Hewitt and Savage in [18] for exchangeable random variables distributed on $X=E \times E \times \ldots, E$ being a compact Hausdorff space.

A noncommutative extension of this result for infinite tensor product $\mathfrak{A}$ of a single $C^{*}$-algebra $\mathfrak{B}$, was given by Størmer in [31], where it

Date: July 20, 2018. 
is shown that the symmetric states on $\mathfrak{A}$ form a simplex whose *weakly closed set of extremal points is made exactly by the product states. Indeed Størmer's theorem, when reduced to abelian $C^{*}$-algebras is nothing else than Hewitt-Savage result.

Other similar characterizations can be found in $[15,19]$ for Boson quantum systems, or in $[3,17]$ in the case of continuous index set. More recently in $[21,22,23]$, the authors got some De Finetti's type theorems in Free Probability, whereas in [1] some results on the structure of symmetric (exchangeable) states on general $C^{*}$-algebras have been obtained.

Besides infinite sequences case, Diaconis and Freedman in [14] obtained a De Finetti theorem for finite sequences of exchangeable random variables. Namely they showed that the first $k$ random variables of a permutation invariant distribution of $n$ random variables can be approximated by a convex combination of $k$ i.i.d. random variables, the error being of order $O\left(k^{2} / n\right)$. This has been the starting point for a recent intensive investigation of finite De Finetti results in Quantum Information Theory and the problem of the Entanglement. Unfortunately, when one generalizes to the tensor product case such a result, it comes out that the approximating error, contrarily to the classical situation, depends on the dimension of the state space (see, e.g. [11] for details). Hence, even if a general extension to infinite dimensional quantum systems does not exist, some precise estimates, independent of the dimension, have been evaluated for many concrete physical classes. In particular, the dependence on the local dimension is removed, for example, when there is a bound on the number of the ways in which the system is measured, or the $n$-particle reduced density matrix is separable [12], when one treats an exponential version of the theorem in the case of coherent states [20], or when one takes orthogonal invariant states [24]. Moreover, an analogous result can be found in the so called Quantum Key Distribution (QKD), when one deals with Gaussian states against general attacks [28], or when one modifies the symmetric hypothesis in a fully compatible way with continuous-variables QKD protocols [25].

Although none of the above mentioned results concern Fermions, nowadays there is a rapidly increasing investigation of properties and models dealing with physical systems describing such particles. We mention for example the following issues which is far to be complete. The investigation of the ground states of lattice systems based on anticommutation annihilators [26]. The introduction and the study of the notion of the product state, and the application to general thermodynamical properties of Fermi lattice systems (see e.g. [4, 5] and 
the references cited therein). The connection with the Markov Property, Quantum Statistical Systems, Quantum Information Theory and Entanglement, of chains of Fermi systems [2, 16]. Disordered systems based on Fermions [6].

Thus, for the natural applications to Quantum Physics and also the general implication in Quantum Probability, it is then natural to address the study of the structure of the symmetric states on the Fermi algebra, that is the CAR $C^{*}$-algebra. Up to the knowledge of the authors, no result concerning the systematic study of this subject is present in literature. This is the object of the present paper. In more details, our purpose consists in:

(1) characterizing the extremal points of the convex set of symmetric states on the CAR algebra,

(2) showing that every symmetric state is the barycenter of a unique maximal Radon measure which is pseudosupported on the extremal states,

(3) proving that the extremal states form a $*$-weakly closed subset (thus the symmetric states are isomorphic to the regular probability measures on a compact Hausdorff space [7]),

(4) determining the type of the von Neumann factors generated by the extremal (i.e. product) states.

One of the main tools in De Finetti theorem for infinite tensor product of $C^{*}$-algebras [31] is the asymptotic Abelianess property with respect to the permutation group. In the CAR algebra this property is not satisfied, because of the anticommutation relations between spatially separated operators. As a consequence, the results relative to the structure of symmetric states in [31] can not be directly imported in our case. Hence, after verifying that the group of permutations which fix all but a finite number of the points in an arbitrary set $J$ (denoted by $\mathbb{P}_{J}$ ), acts as a group of automorphisms on $\operatorname{CAR}(J)$ (the CAR algebra on $J$ ), we establish a result which plays a crucial role in the sequel. Each symmetric (i.e. invariant under the action of the group of permutations above) state on $\operatorname{CAR}(J)$ is even. This property is exploited throughout the paper in order to obtain the results listed above. Indeed, it is used in Theorems 5.3 and 6.1 to prove the equivalence between an extremal symmetric state on $\mathrm{CAR}(J)$ and a product state in the Araki-Moriya sense (the product being constructed starting by a single even state on $\mathbb{M}_{2}(\mathbb{C})$ ), thus reaching point $(1)$.

Further, the even property makes sure that the couple $\left(\mathrm{CAR}(J), \mathbb{P}_{J}\right)$ is $\mathbb{P}_{J}$-Abelian (see Theorem 4.2). This allows to prove that the *weakly compact convex set of symmetric states is a Choquet simplex, 
and, consequently, to obtain the ergodic decomposition presented in (2). The unique decomposing measure is supported on the extremal states when $J$ is countable, and pseudosupported when $J$ is uncountable (see Theorem 5.5 and the final discussion in Section 6).

As a consequence of the above discussion, one has that each symmetric state is (at least in the countable case) a mixture of product states of Araki-Moriya. This statement, which is our main result, can be seen as the extension of De Finetti's theorem to the CAR algebra.

The ending parts of Sections 5 and 6 are aimed to reach points (3) and (4). In particular, some results due to Størmer in [31] and the identification of every even state on $\mathbb{M}_{2}(\mathbb{C})$ with a single point of a closed segment (Lemma 2.2), give the extremal (i.e. product) states are a $*$-weakly closed subset. Furthermore, the very special structure of a product state, allows to achieve point (4), as Propositions 5.7, 5.8 and the relative following discussions.

As stressed above, the Fermi algebra is not asymptotically Abelian with respect to $\mathbb{P}_{J}$, but the even nature of a symmetric state yields a "weak" asymptotic Abelianess property, see (ii) of Theorem 4.1. This result, coupled with the property that $\mathbb{P}_{J}$ acts as a large group of automorphisms (cf. Definition 3.4 and Theorem 4.2), provides some ergodic properties (cf. Proposition 4.3 and Proposition 5.4) for averages and orbits of symmetric states, which, even not used for establishing the main results presented in paper, may have a self-containing interest.

\section{THE CAR ALGEBRA}

Denote by $[a, b]:=a b-b a,\{a, b\}:=a b+b a$, the commutator and anticommutator between elements $a, b$, respectively.

We start by quickly reviewing the basic properties of the Fermion $C^{*}$ algebra, which, due to Pauli Exclusion Principle, is generated by the annihilation and creation operators satisfying the Canonical Anticommutation Relations. Indeed, let $J$ be an arbitrary set. The Canonical Anticommutation Relations (CAR for short) algebra over $J$ is the $C^{*}$ algebra $\operatorname{CAR}(J)$ with the identity $\mathbb{I}$ generated by the set $\left\{a_{j}, a_{j}^{\dagger} \mid j \in J\right\}$ (i.e. the Fermi annihilators and creators respectively), and the relations

$$
\left(a_{j}\right)^{*}=a_{j}^{\dagger},\left\{a_{j}^{\dagger}, a_{k}\right\}=\delta_{j k} \mathbb{I},\left\{a_{j}, a_{k}\right\}=\left\{a_{j}^{\dagger}, a_{k}^{\dagger}\right\}=0, j, k \in J .
$$

The parity automorphism $\Theta$ acts on the generators as

$$
\Theta\left(a_{j}\right)=-a_{j}, \Theta\left(a_{j}^{\dagger}\right)=-a_{j}^{\dagger}, \quad j \in J
$$


and induces on $\operatorname{CAR}(J)$ a $\mathbb{Z}_{2}$-grading. This grading yields $\operatorname{CAR}(J)=$ $\mathrm{CAR}(J)_{+} \oplus \mathrm{CAR}(J)_{-}$, where

$$
\begin{aligned}
& \operatorname{CAR}(J)_{+}:=\{a \in \operatorname{CAR}(J) \mid \Theta(a)=a\}, \\
& \operatorname{CAR}(J)_{-}:=\{a \in \operatorname{CAR}(J) \mid \Theta(a)=-a\} .
\end{aligned}
$$

Elements in $\operatorname{CAR}(J)_{+}$and in $\operatorname{CAR}(J)_{-}$are called even and odd, respectively.

Notice that, by definition,

$$
\operatorname{CAR}(J)=\overline{\operatorname{CAR}_{0}(J)},
$$

where

$$
\operatorname{CAR}_{0}(J):=\bigcup\{\operatorname{CAR}(I) \mid I \subset J \text { finite }\}
$$

is the (dense) subalgebra of the localized elements.

A map $T: \mathcal{A}_{1} \rightarrow \mathcal{A}_{2}$ between $C^{*}$-algebras with $\mathbb{Z}_{2}$-gradings $\Theta_{1}$ and $\Theta_{2}$, is said to be even if it is grading-equivariant:

$$
T \circ \Theta_{1}=\Theta_{2} \circ T \text {. }
$$

The previous definition, applied to states $\varphi \in \mathcal{S}(\mathrm{CAR}(J))$, leads to $\varphi \circ \Theta=\varphi$, that is $\varphi$ is even if and only if it is $\Theta$-invariant.

When the index set $J$ is countable, the $\operatorname{CAR}$ algebra $\operatorname{CAR}(J)$ is isomorphic to the $C^{*}$-infinite tensor product of $J$-copies of $\mathbb{M}_{2}(\mathbb{C})$,

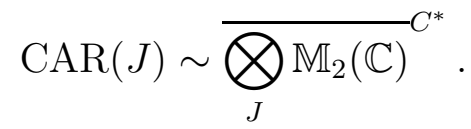

Such an isomorphism is established by a Jordan-Klein-Wigner transformation, as shown in [36], Exercise XIV. We briefly report it for the convenience of the reader. Fix any enumeration $j=1,2, \ldots$ of the set $J$. Let $U_{j}:=a_{j} a_{j}^{\dagger}-a_{j}^{\dagger} a_{j}, j=1,2, \ldots$ Put $V_{0}:=\mathbb{I}, V_{j}:=\prod_{n=1}^{j} U_{n}$, and denote

$$
\begin{aligned}
& e_{11}(j):=a_{j} a_{j}^{\dagger}, \quad e_{12}(j):=V_{j-1} a_{j}, \\
& e_{21}(j):=V_{j-1} a_{j}^{\dagger}, \quad e_{22}(j):=a_{j}^{\dagger} a_{j} .
\end{aligned}
$$

$\left\{e_{k l}(j) \mid k, l=1,2\right\}_{j \in J}$ provides a system of commuting matrix units in $\operatorname{CAR}(\mathbb{N})$. In order to obtain

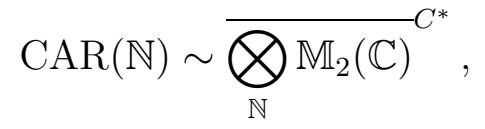

fix any segment $[1, l] \subset \mathbb{N}$ and consider the system of matrix units localized in $r \in \mathbb{N}$

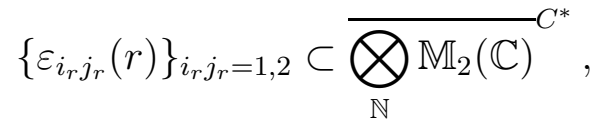


together with the system of matrix units

$$
\left\{e_{i_{r} j_{r}}(r)\right\}_{i_{r} j_{r}=1,2} \subset \mathrm{CAR}(\mathbb{N})
$$

arising from the Jordan-Klein-Wigner construction (2.2). The above isomorphism is simply described by

$$
e_{i_{1} j_{1}}(1) \cdots e_{i_{l} j_{l}}(l) \mapsto \varepsilon_{i_{1} j_{1}}(1) \otimes \cdots \otimes \varepsilon_{i_{l} j_{l}}(l)
$$

for each $i_{k}, j_{k}=1,2, k=1,2, \ldots, l$ and $l \in \mathbb{N}$.

Remark 2.1. Notice that, fixed $r \in \mathbb{N}, e_{i_{r} j_{r}}(r)$ is localized in the segment $[1, r]$ and, moreover, $e_{i_{r} i_{r}}(r)$ is always localized in the site $r$. But $e_{i_{r} j_{r}}(r)$ is not necessarily localized in the site $r$ if $i_{r} \neq j_{r}$.

Anyone of such isomorphisms depends on a predefined order of the countable index set $J$. Thus, it cannot be directly used to investigate the exchangeable properties of the states under consideration.

Thanks to (2.1), CAR $(J)$ has a unique tracial state $\tau$ as the extension of the unique tracial state on $\operatorname{CAR}(I),|I|<+\infty$. Let $I \subset J$ be a finite set and $\varphi \in \mathcal{S}(\mathrm{CAR}(J))$. Then there exists a unique positive element $T \in \operatorname{CAR}(I)$ such that $\varphi\left\lceil_{\operatorname{CAR}(I)}=\tau\left\lceil_{\mathrm{CAR}(I)}(T \cdot)\right.\right.$. The element $T$ is called the adjusted density matrix of $\varphi\lceil\operatorname{CAR}(I)$. For the standard applications to quantum statistical mechanics, one also uses the density matrix w.r.t. the unnormalized trace.

We recall here the description of product state (cf. [5]). We start with the case of finite sets. Namely, let $I_{1}, I_{2} \subset J$ with $\left|I_{1}\right|,\left|I_{2}\right|<\infty$ and $I_{1} \bigcap I_{2}=\emptyset$. Fix $\varphi_{1} \in \mathcal{S}\left(\operatorname{CAR}\left(I_{1}\right)\right), \varphi_{2} \in \mathcal{S}\left(\operatorname{CAR}\left(I_{2}\right)\right)$. If at least one among them is even, then according to Theorem 1 of [5], the product state extension (called product state for short) $\varphi \in \mathcal{S}\left(\operatorname{CAR}\left(I_{1} \cup I_{2}\right)\right.$ ) is uniquely defined. We write, with an abuse of notation, $\varphi=\varphi_{1} \varphi_{2}$. Let $T_{1} \in \mathrm{CAR}\left(I_{1}\right), T_{2} \in \mathrm{CAR}\left(I_{2}\right)$ be the adjusted densities relative to $\varphi_{1} \in \mathcal{S}\left(\mathrm{CAR}\left(I_{1}\right)\right), \varphi_{2} \in \mathcal{S}\left(\mathrm{CAR}\left(I_{2}\right)\right)$, respectively. As at least one among $T_{1}$ and $T_{2}$ is even, $\left[T_{1}, T_{2}\right]=0$ and $T:=T_{1} T_{2}$ is a well defined positive element of $\operatorname{CAR}\left(I_{1} \cup I_{2}\right)$ which is precisely the density matrix of $\varphi=\varphi_{1} \varphi_{2}$. The product state $\varphi \in \mathcal{S}\left(\operatorname{CAR}\left(I_{1} \cup I_{2}\right)\right)$ is even if and only if $\varphi_{1}$ and $\varphi_{2}$ are both even.

Now we pass to the description of the product state on $\operatorname{CAR}(J)$ symbolically written as

$$
\varphi:=\prod_{j \in J} \rho
$$

where $\rho$ is a single even state on $\mathbb{M}_{2}(\mathbb{C}) \sim \operatorname{CAR}(\{j\})$. For $j \in J$ denote $\iota_{j}: \mathbb{M}_{2}(\mathbb{C}) \rightarrow \operatorname{CAR}(J)$ the corresponding embedding. For each finite subset $I:=\left\{j_{1}, \ldots, j_{|I|}\right\} \subset J$, let $\varphi_{I} \in \mathcal{S}(\mathrm{CAR}(I))$ be the product state 
given, on the elementary generators, by

$$
\varphi_{I}\left(\iota_{j_{1}}\left(A_{1}\right) \cdots \iota_{j_{|I|}}\left(A_{|I|}\right)\right)=\prod_{k=1}^{|I|} \rho\left(A_{k}\right),
$$

where $A_{1}, \ldots, A_{|I|} \in \mathbb{M}_{2}(\mathbb{C})$. If $I_{1} \subset I_{2}$, it is immediate to see that $\varphi_{I_{2}}\left\lceil\operatorname{CAR}\left(I_{1}\right)=\varphi_{I_{1}}\right.$. So the direct $\operatorname{limit} \underline{\lim \varphi_{I}}$, when $I \uparrow J$ is a well defined state on the dense $*$-algebra of the localized elements $\operatorname{CAR}_{0}(J)$, which extends by continuity to a state $\varphi$ which is the product state of a single even state $\rho \in \mathbb{M}_{2}(\mathbb{C})$. A necessarily even product state $\varphi=\prod_{J} \rho$ is then uniquely determined by the even state $\rho$, and the next lemma shows that each even state on $\mathbb{M}_{2}(\mathbb{C})$ can be seen as a single point of a closed segment.

Lemma 2.2. For every even state $\rho$ on $\mathbb{M}_{2}(\mathbb{C})$, there exists a unique $\mu \in[0,1]$ such that

$$
\rho\left(\begin{array}{ll}
a & b \\
c & d
\end{array}\right)=\mu a+(1-\mu) d
$$

Proof. By the usual identification of $\operatorname{CAR}(\{j\}), j \in J$ with $\mathbb{M}_{2}(\mathbb{C})$, one sees that for every state $\rho$ on $\mathbb{M}_{2}(\mathbb{C})$, there exists a unique positive matrix $T$ such that $\rho=\tau(T \cdot)$. In particular $T=\left(\begin{array}{ll}\mu & b+\imath f \\ b-\imath f & 1-\mu\end{array}\right)$, with $\mu(1-\mu)-\left(b^{2}+f^{2}\right) \geq 0$, hence $\mu \in[0,1]$. If $\rho$ is even, then $0=\rho\left(\begin{array}{ll}0 & 1 \\ 0 & 0\end{array}\right)=b-\imath f$, hence $b=f=0$. This ends the proof.

We refer to the above $\mu$ and $1-\mu$ as the eigenvalues of the even state $\rho_{\mu}$ on $\mathbb{M}_{2}(\mathbb{C})$, the latter inherited by the $\mathbb{Z}_{2}$-grading arising from $\mathbb{M}_{2}(\mathbb{C}) \sim \operatorname{CAR}(\{j\})$.

Let $\rho_{\mu}$ be an even state as before, and denote $\varphi_{\mu}, \omega_{\mu}$ the correspond-

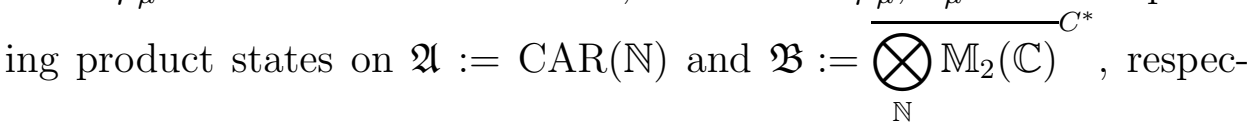
tively. Denote $\gamma: \mathfrak{A} \rightarrow \mathfrak{B}$ the isomorphism described via (2.3).

Lemma 2.3. Under the above notations, for each $\mu \in[0,1]$ we get $\varphi_{\mu}=\omega_{\mu} \circ \gamma$

Proof. By the definition of the product states on $\mathfrak{A}$ and $\mathfrak{B}$, it is enough to check the result for the system of the matrix units. Let $\left\{e_{k l}(j) \mid k, l=\right.$ $1,2\}_{j \in \mathbb{N}},\left\{\varepsilon_{k l}(j) \mid k, l=1,2\right\}_{j \in \mathbb{N}}$ be the canonical systems of matrix units of $\operatorname{CAR}(\mathbb{N})$ and $\overline{\bigotimes_{\mathbb{N}} \mathbb{M}_{2}(\mathbb{C})} C^{*}$ described above. By taking into account 
that $\varphi_{\mu}$ is even and Remark 2.1, we get for the restrictions to any segment $[1, l]$,

$$
\begin{aligned}
\varphi_{\mu}\left(e_{i_{1} j_{1}}(1) \cdots e_{i_{l} j_{l}}(l)\right) & =\varphi_{\mu}\left(e_{i_{1} i_{1}}(1) \cdots e_{i_{l} i_{l}}(l)\right) \delta_{i_{1} j_{1}} \cdots \delta_{i_{l} j_{l}} \\
= & \rho_{\mu}\left(e_{i_{1} i_{1}}(1)\right) \cdots \rho_{\mu}\left(e_{i_{l} i_{l}}(l)\right) \\
= & \omega_{\mu}\left(\varepsilon_{i_{1} i_{1}}(1) \otimes \cdots \otimes \varepsilon_{i_{l} i_{l}}(l)\right) \delta_{i_{1} j_{1}} \cdots \delta_{i_{l} j_{l}} \\
= & \omega_{\mu}\left(\varepsilon_{i_{1} j_{1}}(1) \otimes \cdots \otimes \varepsilon_{i_{l} j_{l}}(l)\right) .
\end{aligned}
$$

3. THE GROUP OF THE PERMUTATIONS AND ITS ACTION ON THE

\section{CAR ALGEBRA}

We firstly present a result, probably known to the experts, crucial in the sequel. Let

$$
G=\bigcup_{\alpha \in A} G_{\alpha}
$$

where $A$ is a directed set, and $G_{\alpha}, \alpha \in A$ are finite subgroups of the group $G$ such that $\alpha<\beta$ implies $G_{\alpha} \subset G_{\beta}$. Consider a unitary representation $\{U(g) \mid g \in G\}$ of $G$ acting on a Hilbert space $\mathcal{H}$. Denote $E_{\alpha}, E$ the selfadjoint projections onto the subspaces of $\mathcal{H}$ consisting of the invariant vectors under the action of $G_{\alpha}$ and $G$, respectively. Of course, the net $\left\{E_{\alpha} \mid \alpha \in A\right\}$ is decreasing. It is straightforward to see (cf. [33], Section 2.17) that it converges in the strong operator topology to a projection

$$
P:=\mathrm{s}-\lim _{\alpha} E_{\alpha} .
$$

In general, $P \geq E$. In addition, it is a standard fact to verify that

$$
E_{\alpha}=\frac{1}{\left|G_{\alpha}\right|} \sum_{g \in G_{\alpha}} U(g) .
$$

Here, we give the analogue of the von Neumann Ergodic Theorem for the case under consideration.

Proposition 3.1. Under the above notations, we get

$$
\mathrm{s}-\lim _{\alpha} E_{\alpha}=E .
$$

Proof. We have only to prove that $P \leq E$. Fix $h \in G$ and $\xi \in \mathcal{H}$ such that

$$
\xi=\lim _{\alpha} \frac{1}{\left|G_{\alpha}\right|} \sum_{g \in G_{\alpha}} U(g) \xi .
$$


As $A$ is a directed set and the sequence of the groups $\left\{G_{\alpha} \mid \alpha \in A\right\}$ is increasing, there exists $\alpha_{h} \in A$ such that $\alpha>\alpha_{h}$ implies $h \in G_{\alpha}$. Then, after a standard change of variables in the sum, we obtain

$$
\begin{aligned}
U(h) \xi & =U(h) \lim _{\alpha} \frac{1}{\left|G_{\alpha}\right|} \sum_{g \in G_{\alpha}} U(g) \xi=U(h) \lim _{\alpha>\alpha_{h}} \frac{1}{\left|G_{\alpha}\right|} \sum_{g \in G_{\alpha}} U(g) \xi \\
& =\lim _{\alpha>\alpha_{h}} \frac{1}{\left|G_{\alpha}\right|} \sum_{g \in G_{\alpha}} U(h g) \xi=\lim _{\alpha>\alpha_{h}} \frac{1}{\left|G_{\alpha}\right|} \sum_{g \in G_{\alpha}} U(g) \xi \\
& =\lim _{\alpha} \frac{1}{\left|G_{\alpha}\right|} \sum_{g \in G_{\alpha}} U(g) \xi=\xi .
\end{aligned}
$$

We now introduce and recall some notations and definitions which will be used throughout the paper. Let $G$ be a group and $\mathfrak{A}$ a $C^{*}$ algebra which we suppose always to be unital. One says that $G$ acts as a group of automorphisms of $\mathfrak{A}$ if there is a representation $\alpha: g \in$ $G \mapsto \alpha_{g} \in \operatorname{Aut}(\mathfrak{A})$. The state $\varphi \in \mathcal{S}(\mathfrak{A})$ is called $G$-invariant if $\varphi=\varphi \circ \alpha_{g}$ for each $g \in G$. The subset $\mathcal{S}_{G}(\mathfrak{A})$ of the $G$-invariant states is $*$-weakly compact in $\mathcal{S}(\mathfrak{A})$, and its extremal points are called ergodic states (w.r.t.the action of $G$ ). For $G$ acting as a group of automorphisms of $\mathfrak{A}$ and a state $\varphi \in \mathcal{S}_{G}(\mathfrak{A}),\left(\pi_{\varphi}, \mathcal{H}_{\varphi}, U_{\varphi}, \Omega_{\varphi}\right)$ is the GNS covariant quadruple canonically associated to $\varphi$ (see, e.g. [8, 35]). If $\left(\pi_{\varphi}, \mathcal{H}_{\varphi}, \Omega_{\varphi}\right)$ is the GNS triple associated to $\varphi$, the unitary representation $U_{\varphi}$ of $G$ on $\mathcal{H}_{\varphi}$ is uniquely determined by

$$
\begin{aligned}
& \pi_{\varphi}\left(\alpha_{g}(A)\right)=U_{\varphi}(g) \pi_{\varphi}(A) U_{\varphi}(g)^{-1}, \\
& U_{\varphi}(g) \Omega_{\varphi}=\Omega_{\varphi}, \quad A \in \mathfrak{A}, g \in G .
\end{aligned}
$$

If $\varphi \in \mathcal{S}_{G}(\mathfrak{A})$, by $\mathfrak{B}_{G}(\varphi):=\left(\mathfrak{Z}_{\varphi}\right)^{\alpha}$ we denote the fixed point algebra of the center

$$
\mathfrak{Z}_{\varphi}:=\pi_{\varphi}(\mathfrak{A})^{\prime \prime} \bigcap \pi_{\varphi}(\mathfrak{A})^{\prime}
$$

under the adjoint action $\operatorname{ad}\left(U_{\varphi}\right)$ of $G$. We will refer to the set

$$
\mathcal{H}_{\varphi}^{G}:=\left\{\xi \in \mathcal{H}_{\varphi} \mid U_{\varphi}(g) \xi=\xi, g \in G\right\}
$$

and $E_{\varphi}$ as the (closed) subspace of $\mathcal{H}_{\varphi}$ of the invariant vectors w.r.t. the action of $G$, and the relative selfadjoint projection onto it, respectively.

Let $(\mathfrak{A}, G)$ be a $C^{*}$-dynamical system as above, together with $\varphi \in$ $\mathcal{S}_{G}(\mathfrak{A})$. The invariant state $\varphi$ is said to be $G$-abelian if all the operators $E_{\varphi} \pi_{\varphi}(\mathfrak{A}) E_{\varphi}$ mutually commute. The $C^{*}$-dynamical system $(\mathfrak{A}, G)$ is $G$-abelian if $\varphi$ is $G$-abelian for each $\varphi \in \mathcal{S}_{G}(\mathfrak{A})$.

Let $J$ be any set. By definition the group of the permutations $\mathbb{P}_{J}$ of $J$ is made by those permutations leaving fixed all the elements of $J$ but 
a finite number of them. Then it is the direct limit of the (sub)groups of the permutations $\mathbb{P}_{I}, I$ running on all the finite subsets of $J$, that is

$$
\mathbb{P}_{J}:=\bigcup\left\{\mathbb{P}_{I} \mid I \subset J \text { finite }\right\} .
$$

It is expected that the group of the permutations $\mathbb{P}_{J}$ acts, in a natural way, as a group of automorphisms of $\operatorname{CAR}(J)$. However this is the case, according to the following

Proposition 3.2. The map $g \in \mathbb{P}_{J} \mapsto a_{g^{-1} j} \in \mathrm{CAR}(J), j \in J$, extends to an action $g \in \mathbb{P}_{J} \mapsto \alpha_{g} \in \operatorname{Aut}(\mathrm{CAR}(J))$ by automorphisms of $\operatorname{CAR}(J)$.

Proof. By a standard argument, we have $\operatorname{CAR}(J) \equiv \mathfrak{A}\left(\ell^{2}(J)\right)$, that is the CAR algebra on $\ell^{2}(J)$ under the notations in Section 5.2.2 of [9]. Our action on the indices is nothing but a unitary action on $\ell^{2}(J)$. This means that $\mathbb{P}_{J}$ acts as a group of Bogoliubov automorphisms of $\operatorname{CAR}(J)$.

We now report a result crucial in the sequel. ${ }^{1}$ Denote $\mathbf{n}:=\{1, \ldots, n\}$ the finite set made of exactly $n$ elements. If $m \leq n, \mathbf{m}$ can be considered, in a canonical way, as a subset of $\mathbf{n}$.

Lemma 3.3. Let $1 \leq m, n<N$. Then, for some constant $c(m, n)$ depending only on $m, n$, we have

$$
\frac{\left|\left\{g \in \mathbb{P}_{\mathbf{N}} \mid \mathbf{m} \cap g \mathbf{n} \neq \emptyset\right\}\right|}{(N-1) !} \leq c(m, n) .
$$

Proof. Set $A:=\left|\left\{g \in \mathbb{P}_{\mathbf{N}} \mid \mathbf{m} \cap g \mathbf{n} \neq \emptyset\right\}^{c}\right|$, and

$$
\begin{aligned}
\Gamma & =(N-m)[\ln (N-m)-1]+(N-n)[\ln (N-n)-1] \\
& -(N-m-n)[\ln (N-m-n)-1]-N(\ln N-1) .
\end{aligned}
$$

It is straightforwardly seen that

$$
\frac{A}{N !}=\frac{(N-m) !(N-n) !}{(N-m-n) ! N !} \approx \sqrt{\frac{(N-m)(N-n)}{N(N-m-n)}} e^{\Gamma},
$$

after using the Stirling formula, for $N \rightarrow+\infty$. By retaining only the leading terms up to the order $1 / N$, we get

$$
\frac{A}{N !} \approx e^{-\frac{m n}{N}} \approx 1-\frac{m n}{N}
$$

\footnotetext{
${ }^{1}$ Compare with the connected estimation in Theorem 13 of [14], concerning the classical case.
} 
which leads to

$$
\frac{\left|\left\{g \in \mathbb{P}_{\mathbf{N}} \mid \mathbf{m} \cap g \mathbf{n} \neq \emptyset\right\}\right|}{N !}=1-\frac{A}{N !} \approx \frac{m n}{N} .
$$

We end the section reporting the definition (cf. [30], Definition 3.3) concerning the action of a group as a Large Group of Automorphisms.

Definition 3.4. Let $g \in G \mapsto \alpha_{g} \in \operatorname{Aut}(\mathfrak{A})$ be an action of a group $G$ on the $C^{*}$-algebra $\mathfrak{A}$. We say that $G$ is represented (or acts) as a large group of automorphisms if, for each selfadjoint $A$ and each $\varphi \in \mathcal{S}_{G}(\mathfrak{A})$

$$
\overline{\operatorname{conv}\left(\left\{\pi_{\varphi}\left(\alpha_{g}(A)\right) \mid g \in G\right\}\right)} \bigcap \pi_{\varphi}(\mathfrak{A})^{\prime} \neq \emptyset .
$$

In the next section we will establish that $\mathbb{P}_{J}$ acts on $\operatorname{CAR}(J)$ as a large group of automorphisms (cf. Theorem 4.2). We underline that this property is not directly used for the main result of the paper concerning the structure of the symmetric (i.e. invariant under the action of $\mathbb{P}_{J}$ ) states. It comes out only for constructing a conditional expectation from the GNS von Neumann algebra of $\operatorname{CAR}(J)$ onto the invariant elements of the center, that allows to obtain some convergence results which may have some interest in general (see Proposition 4.3 and Proposition 5.4).

\section{SYMMETRIC STATES ON THE CAR ALGEBRA}

A state $\varphi \in \mathcal{S}(\mathrm{CAR}(J))$ is called symmetric if it is invariant under the action of the group $\mathbb{P}_{J}$ of all the finite permutations of the set $J$. Following the notation introduced above, $\mathcal{S}_{\mathbb{P}_{J}}(\mathrm{CAR}(J))$ denotes the $*$-weakly compact subset of all the symmetric states of $\operatorname{CAR}(J)$. Furthermore we refer to $\mathcal{E}\left(\mathcal{S}_{\mathbb{P}_{J}}(\mathrm{CAR}(J))\right)$ as the set of all the extremal symmetric states, that is the invariant states which are ergodic w.r.t. the action of $\mathbb{P}_{J}$.

In the section we investigate some of the basic ergodic properties enjoyed by the symmetric states. To this aim, denote $M$ the Cesaro Mean w.r.t. $\mathbb{P}_{J}$, given for a generic object $f(g)$ by

$$
M\{f(g)\}:=\lim _{I \uparrow J} \frac{1}{\left|\mathbb{P}_{I}\right|} \sum_{g \in \mathbb{P}_{I}} f(g),
$$

provided the 1.h.s. exists in the appropriate sense. As usual, $I \subset J$ runs over all the finite parts of $J$.

Theorem 4.1. Let $\varphi \in \mathcal{S}_{\mathbb{P}_{J}}(\mathrm{CAR}(J))$. Then the following assertions hold true. 
(i) The state $\varphi$ is even.

(ii) The state $\varphi$ is asymptotically Abelian in average:

$$
M\left\{\varphi\left(C\left[\alpha_{g}(A), B\right] D\right)\right\}=0, \quad A, B, C, D \in \operatorname{CAR}(J) .
$$

(iii) $\varphi \in \mathcal{E}\left(\mathcal{S}_{\mathbb{P}_{J}}(\mathrm{CAR}(J))\right)$ if and only if it is weakly clustering:

$$
M\left\{\varphi\left(\alpha_{g}(A) B\right)\right\}=\varphi(A) \varphi(B), \quad A, B \in \operatorname{CAR}(J) .
$$

Proof. (i) Let $A$ be localized and odd. Proposition 3.1 gives

$$
\begin{gathered}
\left\{E_{\varphi} \pi_{\varphi}(A) E_{\varphi}, E_{\varphi} \pi_{\varphi}\left(A^{*}\right) E_{\varphi}\right\} \\
=M\left\{E_{\varphi} \pi_{\varphi}(A) U_{\varphi}(g) \pi_{\varphi}\left(A^{*}\right) E_{\varphi}+E_{\varphi} \pi_{\varphi}\left(A^{*}\right) U_{\varphi}(g) \pi_{\varphi}(A) E_{\varphi}\right\} \\
=M\left(E_{\varphi} \pi_{\varphi}\left(\left\{A, \alpha_{g}\left(A^{*}\right)\right\}\right) E_{\varphi}\right)
\end{gathered}
$$

By Lemma 3.3 and the CAR relations, one finds that the quantity above is equal to zero, where the limit in the Cesaro mean is understood in the strong operator topology. ${ }^{2}$ This implies that $E_{\varphi} \pi_{\varphi}(A) E_{\varphi}=0$, that is

$$
\varphi(A)=\left\langle E_{\varphi} \pi_{\varphi}(A) E_{\varphi} \Omega_{\varphi}, \Omega_{\varphi}\right\rangle=0 .
$$

Hence $\varphi$ vanishes on the localized odd elements. Since $\operatorname{CAR}(J) \sim$ $\mathrm{CAR}_{+}(J) \bigoplus \mathrm{CAR}_{-}(J)$ as a Banach space, we can approximate a generic odd element $A$ with a sequence $\left\{A_{n}\right\}_{n \in \mathbb{N}}$ made by odd and localized elements. By the above result, one obtains

$$
\varphi(A)=\varphi\left(\lim _{n} A_{n}\right)=\lim _{n} \varphi\left(A_{n}\right)=0 .
$$

This means that $\varphi$ vanishes on all the odd elements, that is $\varphi$ is even.

(ii) The same computations as before show that, if $A$ or $B$ is even, then

$$
\left[E_{\varphi} \pi_{\varphi}(A) E_{\varphi}, E_{\varphi} \pi_{\varphi}(B) E_{\varphi}\right]=0 .
$$

By a standard approximation argument, we can reduce the matter to localized elements. Fix $A$ even. Proposition 3.1, Lemma 3.3 and (4.1), give

$$
\begin{aligned}
& M\left\{\varphi\left(C \alpha_{g}(A) B D\right)\right\}=M\left\{\varphi\left(\alpha_{g}(A) C B D\right)\right\} \\
= & \left\langle\pi_{\varphi}(A) E_{\varphi} \pi_{\varphi}(C B D) \Omega_{\varphi}, \Omega_{\varphi}\right\rangle=\left\langle\pi_{\varphi}(C B D) E_{\varphi} \pi_{\varphi}(A) \Omega_{\varphi}, \Omega_{\varphi}\right\rangle \\
= & M\left\{\varphi\left(C B D \alpha_{g}(A)\right)\right\}=M\left\{\varphi\left(C B \alpha_{g}(A) D\right)\right\} .
\end{aligned}
$$

By considering $A$ odd and splitting $C, D$ in their even and odd parts, the result is reached by similar computations as above.

\footnotetext{
${ }^{2}$ The analogous case based on the spatial translations has been treated in [9], Example 5.2.21, where the particular form of the action of $\mathbb{Z}^{d}$ on $\operatorname{CAR}\left(\mathbb{Z}^{d}\right)$ as the shift, allows to reach directly the result without using Lemma 3.3.
} 
(iii) The weak clustering condition is equivalent to $\operatorname{dim}\left(\mathcal{H}_{\varphi}^{\mathbb{P}_{J}}\right)=1$, and it is immediate to show that implies ergodicity (see [29], Proposition 3.1.10). The converse assertion follows from Theorem 4.2, that is $\operatorname{CAR}(J)$ is $\mathbb{P}_{J}$-abelian (see [29], Proposition 3.1.12).

Theorem 4.2. The group $\mathbb{P}_{J}$ acts as a Large Groups of Automorphisms on $\mathrm{CAR}(J)$, and the $C^{*}$-dynamical system $\left(\mathrm{CAR}(J), \mathbb{P}_{J}, \alpha\right)$ is $\mathbb{P}_{J}$-abelian.

Proof. By taking into account (4.1) and (i) of Theorem 4.1, we conclude that $\left(\mathrm{CAR}(J), \mathbb{P}_{J}\right)$ is $\mathbb{P}_{J}$-Abelian. Fix an arbitrary integer $n \in \mathbb{N}$, $A, B_{1}, \ldots, B_{n}, C \in \operatorname{CAR}(J)$ and $\varphi \in \mathcal{S}_{\mathbb{P}_{J}}(\operatorname{CAR}(J))$. Now, by (ii) of Theorem 4.1, for each $\varepsilon>0$ there exists a finite set $I \subset J$ such that, for any $k=1, \ldots, n$,

$$
\begin{aligned}
& \left|\varphi\left(C^{*}\left[\left(\sum_{g \in \mathbb{P}_{I}} \frac{\alpha_{g}(A)}{\left|\mathbb{P}_{I}\right|}\right), B_{k}\right] C\right)\right| \\
= & \left|\frac{1}{\left|\mathbb{P}_{I}\right|} \sum_{g \in \mathbb{P}_{I}} \varphi\left(C^{*}\left[\alpha_{g}(A), B_{k}\right] C\right)\right|<\varepsilon .
\end{aligned}
$$

This leads to the assertion by Theorem 3.5 of [30].

As $\mathbb{P}_{J}$ is acting on $\mathrm{CAR}(J)$ as a large group of automorphisms, by Theorem 3.1 of [30], for each state $\varphi \in \mathcal{S}_{\mathbb{P}_{J}}(\mathrm{CAR}(J))$ there exists a conditional expectation

$$
\Phi_{\varphi}: \pi_{\varphi}(\mathrm{CAR}(J))^{\prime \prime} \rightarrow \mathfrak{B}_{\mathbb{P}_{J}}(\varphi)
$$

of the von Neumann algebra $\pi_{\varphi}(\operatorname{CAR}(J))^{\prime \prime}$ onto $\mathfrak{B}_{\mathbb{P}_{J}}(\varphi)$. As the state $\varphi$ is asymptotically Abelian in average (cf. (ii) of Theorem 4.1) we prove the following result which, even if is not used in the sequel, may have an interest in itself.

Proposition 4.3. Let $\varphi \in \mathcal{S}_{\mathbb{P}_{J}}(\mathrm{CAR}(J))$ and $A \in \mathrm{CAR}(J)$. Then

$$
\mathrm{w}-\lim _{I \uparrow J} \frac{1}{\left|\mathbb{P}_{I}\right|} \sum_{g \in \mathbb{P}_{I}} U_{\varphi}(g) \pi_{\varphi}(A) U_{\varphi}(g)^{-1}=\Phi_{\varphi}\left(\pi_{\varphi}(A)\right) .
$$

Proof. Let $\left\{I_{\beta}\right\} \subset\{I\}$ any subnet of the Cauchy net $\{I\}$ of all the finite subsets $I \subset J$ such that $\frac{1}{\left|\mathbb{P}_{I_{\beta}}\right|} \sum_{g \in \mathbb{P}_{I_{\beta}}} U_{\varphi}(g) \pi_{\varphi}(A) U_{\varphi}(g)^{-1}$ converges in the weak operator topology, which exists by compactness. Fix an arbitrary $h \in \mathbb{P}_{J}$. Then there exists a $\beta_{h}$ such that $I \supset I_{\beta_{h}}$ implies 
$h \in \mathbb{P}_{I}$. We get

$$
\begin{aligned}
& \mathrm{w}-\lim _{I_{\beta}} U_{\varphi}(h)\left(\frac{1}{\left|\mathbb{P}_{I_{\beta}}\right|} \sum_{g \in \mathbb{P}_{I_{\beta}}} U_{\varphi}(g) \pi_{\varphi}(A) U_{\varphi}(g)^{-1}\right) U_{\varphi}(h)^{-1} \\
= & \mathrm{w}-\lim _{I_{\beta} \supset I_{\beta_{h}}} \frac{1}{\left|\mathbb{P}_{I_{\beta}}\right|} \sum_{g \in \mathbb{P}_{I_{\beta}}} U_{\varphi}(h g) \pi_{\varphi}(A) U_{\varphi}(h g)^{-1} \\
= & \mathrm{w}-\lim _{I_{\beta} \supset I_{\beta_{h}}} \frac{1}{\left|\mathbb{P}_{I_{\beta}}\right|} \sum_{g \in \mathbb{P}_{I_{\beta}}} U_{\varphi}(g) \pi_{\varphi}(A) U_{\varphi}(g)^{-1} \\
= & \mathrm{w}-\lim _{I_{\beta}} \frac{1}{\left|\mathbb{P}_{I_{\beta}}\right|} \sum_{g \in \mathbb{P}_{I_{\beta}}} U_{\varphi}(g) \pi_{\varphi}(A) U_{\varphi}(g)^{-1}
\end{aligned}
$$

Thus, each weak limit point of the Cesaro net on the l.h.s. of (4.2) is invariant. By using the asymptotic Abelianess property (ii) of Theorem 4.1 , and arguing as in the proof of Lemma 5.3 of [30], one shows that the limit above is in $\mathfrak{Z}_{\varphi}$. Then it belongs to $\mathfrak{B}_{\mathbb{P}_{J}}(\varphi)$. But $\mathfrak{B}_{\mathbb{P}_{J}}(\varphi)$ can contain at most one of such limit points since $\mathbb{P}_{J}$ acts on $\operatorname{CAR}(J)$ as a large group of automorphisms (cf. proof of Theorem 3.1 in [30]). As a consequence, the limit is precisely $\Phi_{\varphi}\left(\pi_{\varphi}(A)\right)$.

\section{THE STRUCTURE OF THE SYMMETRIC STATES: DE FINETTI}

\section{THEOREM}

The present and the following sections are mainly concerned with the characterization of the extremal symmetric states. In particular here we consider the case in which $J$ is countable, hence $J \equiv \mathbb{N}$. We preliminary report the definition of the sequence of permutations $\left\{g_{n}\right\}_{n \in \mathbb{N}}$ in [31] given by

$$
g_{n}(k):= \begin{cases}2^{n-1}+k & \text { if } 1 \leq k \leq 2^{n-1} \\ k-2^{n-1} & \text { if } 2^{n-1}<k \leq 2^{n}, \\ k & \text { if } 2^{n}<k\end{cases}
$$

Definition 5.1. A state $\varphi \in \mathcal{S}(\mathrm{CAR}(\mathbb{N}))$ is said to be strongly clustering if, for every $A, B \in \mathrm{CAR}(\mathbb{N})$

$$
\lim _{n} \varphi\left(\alpha_{g_{n}}(A) B\right)=\varphi(A) \varphi(B) .
$$

Lemma 5.2. If $\varphi \in \mathcal{S}_{\mathbb{P}_{\mathbb{N}}}(\mathrm{CAR}(\mathbb{N}))$ is extremal, then for each $A \in$ $\operatorname{CAR}(\mathbb{N})$

$$
\mathrm{w}-\lim _{n}\left[\pi_{\varphi}\left(\alpha_{g_{n}}(A)\right) \Omega_{\varphi}\right]=\varphi(A) \Omega_{\varphi}
$$


Proof. By a standard approximation argument, we can reduce the matter to $A \in \mathrm{CAR}_{0}(\mathbb{N})$. Let $g \in \mathbb{P}_{\mathbb{N}}$. As shown in the proof of Lemma 2.6 of [31], there exists $n_{A, g}$ such that $n>n_{A, g}$ implies $\alpha_{g g_{n}}(A)=\alpha_{g_{n}}(A)$. This means that any weak limit point (which exists by compactness) of the sequence $\left\{\pi_{\varphi}\left(\alpha_{g_{n}}(A)\right) \Omega_{\varphi}\right\}$ is an invariant vector under the action of $\mathbb{P}_{\mathbb{N}}$, that is it belongs to $\mathcal{H}_{\varphi}^{\mathbb{P}_{\mathbb{N}}}$. Let

$\xi:=\mathrm{w}-\lim _{k} U_{\varphi}\left(g_{n_{\xi}(k)}\right) \pi_{\varphi}(A) U_{\varphi}\left(g_{n_{\xi}(k)}\right)^{-1} \Omega_{\varphi} \equiv \mathrm{w}-\lim _{k} \pi_{\varphi}\left(\alpha_{g_{n_{\xi}(k)}}(A)\right) \Omega_{\varphi}$

be one of such limit points. Since $\xi$ is a vector in $\mathcal{H}_{\varphi}^{\mathbb{P}_{\mathbb{N}}}$ and $\varphi$ is extremal, by (iii) of Theorem 4.1, one has $\xi=\Gamma(A, \xi) \Omega_{\varphi}$. By using (5.1), we obtain

$$
\begin{aligned}
\Gamma(A, \xi) & =\left\langle\lim _{k} U_{\varphi}\left(g_{n_{\xi}(k)}\right) \pi_{\varphi}(A) U_{\varphi}\left(g_{n_{\xi}(k)}\right)^{-1} \Omega_{\varphi}, \Omega_{\varphi}\right\rangle \\
& =\lim _{k}\left\langle\pi_{\varphi}\left(\alpha_{g_{n_{\xi}(k)}}(A) \Omega_{\varphi}, \Omega_{\varphi}\right\rangle=\varphi(A) .\right.
\end{aligned}
$$

Namely, there is only one of such weak limit points in $\mathcal{H}_{\varphi}$, which is $\varphi(A) \Omega_{\varphi}$

Theorem 5.3. Let $\varphi \in \mathcal{S}_{\mathbb{P}_{\mathbb{N}}}(\mathrm{CAR}(\mathbb{N}))$. Then the following are equivalent.

(i) $\varphi \in \mathcal{E}\left(\mathcal{S}_{\mathbb{P}_{\mathbb{N}}}(\operatorname{CAR}(\mathbb{N}))\right)$,

(ii) $\varphi$ is strongly clustering,

(iii) $\varphi=\prod_{\mathbb{N}} \rho$ for some even state $\rho \in \mathbb{M}_{2}(\mathbb{C})$.

Proof. $(i) \Rightarrow($ ii $)$ Suppose $\varphi$ is extremal and take $A, B \in \operatorname{CAR}(\mathbb{N})$. Then by Lemma 5.2 , we get

$$
\lim _{n} \varphi\left(A \alpha_{g_{n}}(B)\right)=\lim _{n}\left\langle\pi_{\varphi}\left(\alpha_{g_{n}}(B)\right) \Omega_{\varphi}, \pi_{\varphi}\left(A^{*}\right) \Omega_{\varphi}\right\rangle=\varphi(A) \varphi(B),
$$

that is $\varphi$ is strongly clustering.

(ii $) \Rightarrow(i)$ Choose a vector $\xi \perp \Omega_{\varphi}$ belonging to $\mathcal{H}_{\varphi}^{\mathbb{P}_{N}}$, and fix $\varepsilon>0$. Then there exists $B \in \mathrm{CAR}(\mathbb{N})$ such that $\left\|\xi-\pi_{\varphi}(B) \Omega_{\varphi}\right\|<\varepsilon / 2$. Let $A \in \operatorname{CAR}(\mathbb{N})$ such that $\left\|\pi_{\varphi}(A)\right\| \leq 1$. We get

$$
\begin{aligned}
& \left|\left\langle\xi, \pi_{\varphi}(A) \Omega_{\varphi}\right\rangle\right|=\left|\left\langle U_{\varphi}\left(g_{n}\right) \pi_{\varphi}\left(A^{*}\right) U_{\varphi}\left(g_{n}\right)^{-1} \xi, \Omega_{\varphi}\right\rangle\right| \\
\leq & \left|\left\langle U_{\varphi}\left(g_{n}\right) \pi_{\varphi}\left(A^{*}\right) U_{\varphi}\left(g_{n}\right)^{-1} \pi_{\varphi}(B) \Omega_{\varphi}, \Omega_{\varphi}\right\rangle\right|+\varepsilon / 2=\left|\varphi\left(\alpha_{g_{n}}\left(A^{*}\right) B\right)\right|+\varepsilon / 2 .
\end{aligned}
$$

Suppose now $\varphi$ is strongly clustering. By taking the limit for $n \rightarrow \infty$ on both sides, we get

$$
\left|\left\langle\xi, \pi_{\varphi}(A) \Omega_{\varphi}\right\rangle\right| \leq\left|\varphi\left(A^{*}\right)\right||\varphi(B)|+\varepsilon / 2<\varepsilon .
$$

As $\varepsilon>0$ is arbitrary and $\Omega_{\varphi}$ is cyclic for $\pi_{\varphi}(\mathrm{CAR}(\mathbb{N}))$, we get $\xi=0$. This means that $\mathcal{H}_{\varphi}^{\mathbb{P}_{\mathbb{N}}}$ is one dimensional, which implies (and it is indeed equivalent by Proposition 3.1.12 of [29]) that $\varphi$ is extremal. 
Obviously, if $\varphi$ is a product state of a single state as in (iii), then it is strongly clustering. Suppose now that $(i i)$ holds true. After fixing $j \in \mathbb{N}$, we start by identifying $\operatorname{CAR}(\{j\})$ with $\mathbb{M}_{2}(\mathbb{C})$ and, as usual, denoting $\iota_{j}: \mathbb{M}_{2}(\mathbb{C}) \rightarrow \mathrm{CAR}(\mathbb{N})$ the related embedding.

By Theorem 1 of [5], any product state is uniquely determined by the product of the values of the state on the generators. Hence, it is enough to check, for each $n \in \mathbb{N}$ and $A_{1}, \ldots, A_{n} \in \mathbb{M}_{2}(\mathbb{C})$,

$$
\varphi\left(\iota_{1}\left(A_{1}\right) \cdots \iota_{n}\left(A_{n}\right)\right)=\prod_{j=1}^{n} \rho\left(A_{j}\right),
$$

The proof now proceeds as in Theorem 2.7 of [31]. We give the details with the appropriate modifications. Define for $j \in \mathbb{N}, \rho_{j}(A):=$ $\varphi\left(\iota_{j}(A)\right)$. As $\varphi$ is even (cf. Theorem 4.1.(i)), all the $\rho_{j}$ are even. In addition, for $i, j \in \mathbb{N}, \rho_{i}=\rho_{j}=: \rho$ as $\varphi$ is symmetric. Equation (5.2) can be achieved by an induction procedure. Indeed, for $n=1$ it follows immediately, so we suppose it holds true till $n-1$. Fix $\varepsilon>0$. By using the inductive hypothesis and the strong clustering property, we get

$$
\begin{aligned}
& \text { (5.3) }\left|\varphi\left(\iota_{1}\left(A_{1}\right) \cdots \iota_{n}\left(A_{n-1}\right) \alpha_{g_{m}}\left(\iota_{n}\left(A_{n}\right)\right)\right)-\prod_{j=1}^{n} \rho\left(A_{j}\right)\right| \\
& =\left|\varphi\left(\iota_{1}\left(A_{1}\right) \cdots \iota_{n}\left(A_{n-1}\right) \alpha_{g_{m}}\left(\iota_{n}\left(A_{n}\right)\right)\right)-\varphi\left(\iota_{1}\left(A_{1}\right) \cdots \iota_{n}\left(A_{n-1}\right)\right) \rho\left(A_{n}\right)\right|<\varepsilon
\end{aligned}
$$

for some $m>n$. Choose now a permutation $g \in \mathbb{P}_{\mathbb{N}}$ such that $g(j)=j$ if $1 \leq j<n$ and $g(n)=g_{m}(n)$. Then

$$
\begin{aligned}
\varphi\left(\iota_{1}\left(A_{1}\right) \cdots \iota_{n}\left(A_{n}\right)\right) & =\varphi\left(\alpha_{g}\left(\iota_{1}\left(A_{1}\right) \cdots \iota_{n}\left(A_{n}\right)\right)\right) \\
& =\varphi\left(\iota_{1}\left(A_{1}\right) \cdots \iota_{n}\left(A_{n-1}\right) \alpha_{g_{m}}\left(\iota_{n}\left(A_{n}\right)\right)\right)
\end{aligned}
$$

which, combined with (5.3), leads to the assertion as $\varepsilon>0$ is arbitrary.

When a state is extremal among the symmetric ones, the result of Proposition 4.3 can be strengthened as we see in the following proposition, inserted for the sake of completeness.

Proposition 5.4. If $\varphi \in \mathcal{E}\left(\mathcal{S}_{\mathbb{P}_{\mathbb{N}}}(\mathrm{CAR}(\mathbb{N}))\right)$. Then for each $A \in \mathrm{CAR}(\mathbb{N})$,

$$
\mathrm{w}-\lim _{n} U_{\varphi}\left(g_{n}\right) \pi_{\varphi}(A) U_{\varphi}\left(g_{n}\right)^{-1}=\varphi(A) \mathbb{I}=\Phi_{\varphi}\left(\pi_{\varphi}(A)\right) .
$$

Proof. By a standard approximation argument, it is enough to consider $\xi=\pi_{\varphi}(B) \Omega_{\varphi}, \eta=\pi_{\varphi}\left(C^{*}\right) \Omega_{\varphi}$, and reduce the matter to $A, B, C \in$ $\mathrm{CAR}_{0}(\mathbb{N})$. Let $A=A_{+}+A_{-}, B=B_{+}+B_{-}$the split of $A, B$ into the even and odd part. By Theorem 5.3, it is enough to assume 
that $\varphi$ is strongly clustering. As $\varphi$ is even, by using the standard (anti)commutation relations, we get

$$
\begin{aligned}
& \lim _{n}\left\langle U_{\varphi}\left(g_{n}\right) \pi_{\varphi}(A) U_{\varphi}\left(g_{n}\right)^{-1} \xi, \eta\right\rangle=\lim _{n} \varphi\left(C \alpha_{g_{n}}(A) B\right) \\
= & \lim _{n} \varphi\left(C B \alpha_{g_{n}}\left(A_{+}\right)\right)+\lim _{n} \varphi\left(C B_{+} \alpha_{g_{n}}\left(A_{-}\right)\right)-\lim _{n} \varphi\left(C B_{-} \alpha_{g_{n}}\left(A_{-}\right)\right) \\
= & \varphi(C B) \varphi\left(A_{+}\right)+\varphi\left(C B_{+}\right) \varphi\left(A_{-}\right)-\varphi\left(C B_{-}\right) \varphi\left(A_{-}\right) \\
= & \varphi(C B) \varphi\left(A_{+}\right)+\varphi\left(C B_{+}\right) \varphi\left(A_{-}\right) \\
= & \varphi(C B) \varphi\left(A_{+}\right)+\varphi\left(C B_{+}\right) \varphi\left(A_{-}\right)+\varphi\left(C B_{-}\right) \varphi\left(A_{-}\right) \\
= & \varphi(C B) \varphi(A)=\langle(\varphi(A) \mathbb{I}) \xi, \eta\rangle .
\end{aligned}
$$

This means that the above weak limit is in $\mathfrak{B}_{\mathbb{P}_{\mathbb{N}}}(\varphi)$. But, as observed in the proof of (4.2), it is nothing else than $\Phi_{\varphi}\left(\pi_{\varphi}(A)\right)$.

Notice that, in the case of the $C^{*}$-tensor product (i.e. when the system is asymptotically Abelian in norm) (5.4) is satisfied for each symmetric state, see [31], Lemma 2.6. In our situation, we have merely the expected average property (4.2), and (5.4) is satisfied only for extremal symmetric states.

Having characterized the extremal symmetric states as the ArakiMoriya product states, we are interested in the ergodic decomposition of an invariant state under the action of the permutation group. Namely, we want to express every symmetric state as the barycenter of a unique (maximal) Radon probability measure on $\mathcal{S}_{\mathbb{P}_{\mathbb{N}}}(\mathrm{CAR}(\mathbb{N})$ ) whose support is $\mathcal{E}\left(\mathcal{S}_{\mathbb{P}_{\mathbb{N}}}(\mathrm{CAR}(\mathbb{N}))\right)$. The existence of such a measure is granted, since $\mathcal{S}_{\mathbb{P}_{\mathbb{N}}}(\mathrm{CAR}(\mathbb{N})$ ) is metrizable (see [8], Proposition 4.1.3 and Theorem 4.1.11). It is unique if and only if $\mathcal{S}_{\mathbb{P}_{\mathbb{N}}}(\mathrm{CAR}(\mathbb{N}))$ is a Choquet simplex (see [8], Theorem 4.1.15). This is our case, as we see in the following result.

Theorem 5.5. $\mathcal{S}_{\mathbb{P}_{\mathbb{N}}}(\mathrm{CAR}(\mathbb{N}))$ is a Choquet simplex. Then, for each $\varphi \in \mathcal{S}_{\mathbb{P}_{\mathbb{N}}}(\mathrm{CAR}(\mathbb{N}))$ there exists a unique maximal Radon probability measure $\mu$ on $\mathcal{S}_{\mathbb{P}_{\mathbb{N}}}(\mathrm{CAR}(\mathbb{N}))$ such that

$$
\varphi(A)=\int \psi(A) d \mu(\psi), \quad A \in \operatorname{CAR}(\mathbb{N}),
$$

and $\mu\left(\mathcal{E}\left(\mathcal{S}_{\mathbb{P}_{\mathbb{N}}}(\mathrm{CAR}(\mathbb{N}))\right)=1\right.$.

Proof. By Theorem 4.2, $\left(\mathrm{CAR}(\mathbb{N}), \mathbb{P}_{\mathbb{N}}\right)$ is $\mathbb{P}_{\mathbb{N}}-$ Abelian, then, as a consequence of Theorem 3.1.14 of [29], $\mathcal{S}_{\mathbb{P}_{\mathbb{N}}}(\mathrm{CAR}(\mathbb{N}))$ is a Choquet simplex. The last part is a rephrasing of the above discussion.

The property for the set of extremal states to be $*$-weakly closed implies a nice result. In fact, in [7] it is shown that a simplex with 
closed boundary is affinely isomorphic to the probability measures on a compact Hausdorff space. These facts are stated in the following proposition, whose proof, based on Lemma 2.2, Theorem 5.3 and arguments analogous to those developed in Theorem 2.8 of [31], is left to the reader.

Proposition 5.6. The Choquet simplex $\mathcal{S}_{\mathbb{P}_{\mathbb{N}}}(\mathrm{CAR}(\mathbb{N}))$ has a $*$-weakly closed boundary and is affinely isomorphic to the probability measures on a closed interval.

Put $\mathfrak{A}:=\operatorname{CAR}(\mathbb{N})$. Let $\varphi \in \mathcal{S}(\mathfrak{A})$. Obviously, $\pi_{\varphi}(\mathfrak{A})^{\prime \prime}$ is a hyperfinite von Neumann algebra. A state $\varphi$ on a $C^{*}$-algebra $\mathfrak{A}$ is called factor state if the double commutant $\pi_{\varphi}(\mathfrak{A})^{\prime \prime}$ of $\pi_{\varphi}(\mathfrak{A})$ is a factor. The state $\varphi$ is said of type $X$ if $\pi_{\varphi}(\mathfrak{A})^{\prime \prime}$ is of type $X$, where $X=\mathrm{I}_{\infty}, \mathrm{II}_{1}, \mathrm{II}_{\infty}$, III or $\mathrm{III}_{\lambda}, \lambda \in[0,1]$ according to the Connes' classification of the type III factors $[10]$.

Proposition 5.7. Let $\rho_{\mu}$ be a even state in $\mathbb{M}_{2}(\mathbb{C})$ with eigenvalues $\mu$ and $1-\mu$, and $\varphi_{\mu}:=\prod_{\mathbb{N}} \rho_{\mu}$ the corresponding product state on $\mathrm{CAR}(\mathbb{N})$. Then

(1) $\varphi_{\mu}$ is a factor state of type $\mathrm{I}_{\infty}$ if and only if $\mu=0$ or $\mu=1$.

(2) $\varphi_{\mu}$ is a factor state of type $\mathrm{II}_{1}$ if and only if $\mu=1 / 2$.

(3) $\pi_{\varphi_{\mu}}(\mathfrak{A})^{\prime \prime}$ is of type $\mathrm{III}_{\lambda}$ if and only if $0<\mu<1 / 2$ and $\lambda=\frac{\mu}{1-\mu}$, or $1 / 2<\mu<1$ and $\lambda=\frac{1-\mu}{\mu}$.

Proof. By Lemma 2.3, we get (under the same notations) $\pi_{\varphi_{\mu}}(\mathfrak{A})^{\prime \prime} \sim$ $\pi_{\omega_{\mu}}(\mathfrak{B})^{\prime \prime}$. But the $\pi_{\omega_{\mu}}(\mathfrak{B})^{\prime \prime}$ are factors whose type is determined by the ratio between the smallest and the largest eigenvalue of the trace operator describing $\rho_{\mu}$, according to the three possibilities listed above in the statement. The reader is referred to A.17 of [32] and the references cited therein.

Notice that we do not have the type $\mathrm{II}_{\infty}$, and furthermore, the type $\mathrm{II}_{1}$ occurs only for $\varphi_{1 / 2}$. Thus, the latter gives rise the trivial face made of a singleton. ${ }^{3}$ The cases of the portions corresponding to $\mathrm{I}_{\infty}$ and III are covered by the next result. Recall that a face of a given simplex $K$ is a convex subset $F$ of $K$ such that, if $\chi \in F, \psi \in K$ and for $\mu>0$, $\psi \leq \mu \chi$, implies $\psi \in F$. The proof of the forthcoming proposition follows mutatis mutandis the lines of the analogous Lemma 2.9 in [31]. The details are left to the reader.

\footnotetext{
${ }^{3}$ The $\mathrm{II}_{\infty}$ might appear for the more general situation investigated in [16], where the regrouped CAR algebra with $2 d$ generators is attacked to each site.
} 
Proposition 5.8. If $X$ denotes $\mathrm{I}_{\infty}$ or $\mathrm{III}$, and

$$
\mathcal{S}_{\mathbb{P}_{\mathbb{N}}}(\operatorname{CAR}(\mathbb{N}))_{X}:=\left\{\varphi \in \mathcal{S}_{\mathbb{P}_{\mathbb{N}}}(\operatorname{CAR}(\mathbb{N})) \mid \varphi \text { is of type } X\right\},
$$

then $\mathcal{S}_{\mathbb{P}_{\mathbb{N}}}(\operatorname{CAR}(\mathbb{N}))_{X}$ is a face of $\mathcal{S}_{\mathbb{P}_{\mathbb{N}}}(\mathrm{CAR}(\mathbb{N}))$.

Recalling that the boundary of an arbitrary nontrivial compact convex $K$ is nonvoid by the Krein-Milman Theorem, by Propositions 5.6 and 5.7 , one has that $\mathcal{E}\left(\mathcal{S}_{\mathbb{P}_{\mathbb{N}}}(\mathrm{CAR}(\mathbb{N}))_{\text {III }}\right)$ consists of two open connected components of $\mathcal{E}\left(\mathcal{S}_{\mathbb{P}_{\mathbb{N}}}(\mathrm{CAR}(\mathbb{N}))\right), \mathcal{E}\left(\mathcal{S}_{\mathbb{P}_{\mathbb{N}}}(\mathrm{CAR}(\mathbb{N}))_{\mathrm{I}_{\infty}}\right)$ is given by two states, that is the pure states, whereas $\mathcal{E}\left(\mathcal{S}_{\mathbb{P}_{\mathbb{N}}}(\mathrm{CAR}(\mathbb{N}))_{\mathrm{II}_{1}}\right)$ is made by one state, that is the unique trace.

We end the section with a brief sketch on the extension of the situation in Proposition 5.8 to more general cases and leave further details to the reader.

Let $\mathfrak{A}$ be a separable $C^{*}$-algebra and $E \in(0,1)$ be a Borel set. Fix a state $\varphi \in \mathcal{S}(\mathfrak{A})$ and put $M:=\pi_{\varphi}(\mathfrak{A})^{\prime \prime}$. Let

$$
M=\int_{\Gamma}^{\otimes} M_{\gamma} \mathrm{d} \nu(g)
$$

be its direct integral decomposition into von Neumann factors. Here, $(\Gamma, \nu)$ is a standard probability measure space which can be chosen as $\left(\operatorname{spec}\left(\mathfrak{Z}_{\varphi}\right), \nu_{\omega\left\lceil_{\mathfrak{Z}_{\varphi}}\right.}\right), \operatorname{spec}\left(\mathfrak{Z}_{\varphi}\right)$ and $\omega$ being the spectrum of $\mathfrak{Z}_{\varphi}$, and a faithful normal state on $M$ respectively, see e.g. [29, 35].

We say that the state $\varphi$ is of type $X_{E}$ if $M$ contains only type $\mathrm{III}_{\lambda}$ factors for some $\lambda \in E$ in its direct integral decomposition (5.5). Let $\Gamma_{E} \subset \Gamma$ be the subset made of the $\gamma$ such that $M_{\gamma}$ is a type $\mathrm{III}_{\lambda}$ factor for some $\lambda \in E$. By Theorem 2.2 of [34] (see also (ii) in Theorem 21.2 of [27]), $\Gamma_{E}$ is a $\nu$-measurable set. The fact that $\varphi$ is of type $X_{E}$ simply means that $\nu\left(\Gamma_{E}\right)=1$. Put

$$
\mathcal{S}_{\mathbb{P}_{\mathbb{N}}}(\mathfrak{A})_{X_{E}}:=\left\{\varphi \in \mathcal{S}_{\mathbb{P}_{\mathbb{N}}}(\mathfrak{A}) \mid \varphi \text { is of type } X_{E}\right\} .
$$

Remark 5.9. By using the same lines as in Lemma 2.9 of [31], we can show that $\mathcal{S}_{\mathbb{P}_{\mathbb{N}}}(\operatorname{CAR}(\mathbb{N}))_{X_{E}}$ is a face of $\mathcal{S}_{\mathbb{P}_{\mathbb{N}}}(\operatorname{CAR}(\mathbb{N}))$.

In fact, let $\varphi=\lambda \varphi_{1}+(1-\lambda) \varphi_{2}, \lambda \in[0,1]$ be a convex combination of states in $\mathcal{S}_{\mathbb{P}_{\mathbb{N}}}(\mathrm{CAR}(\mathbb{N}))_{X_{E}}$. As in the proof of Lemma 2.9 of [31], we find $P_{1}, P_{2} \in \mathfrak{Z}_{\varphi}$ such that

$$
\pi_{\varphi_{k}}(\mathfrak{A})^{\prime \prime}=P_{k} \pi_{\varphi}(\mathfrak{A})^{\prime \prime}, \quad k=1,2 .
$$

Of course, $P_{1} \pi_{\varphi}(\mathfrak{A})^{\prime \prime}$ contains only type $\mathrm{III}_{\lambda}$ factors, $\lambda \in E$, in its direct integral factor decomposition (5.5). The same happens to $\left(P_{2}-\right.$ $\left.P_{1} P_{2}\right) \pi_{\varphi}(\mathfrak{A})^{\prime \prime}$ as $P_{2}-P_{1} P_{2} \leq P_{2}$. Since one can show that $P_{1}+P_{2}-$ $P_{1} P_{2}=I$, it follows that $\varphi$ is of type $X_{E}$. So $\mathcal{S}_{\mathbb{P}_{\mathbb{N}}}(\operatorname{CAR}(\mathbb{N}))_{X_{E}}$ is closed under convex combinations. Let now take $\omega \in \mathcal{S}_{\mathbb{P}_{\mathbb{N}}}(\mathrm{CAR}(\mathbb{N}))$, 
$\varphi \in \mathcal{S}_{\mathbb{P}_{\mathbb{N}}}(\operatorname{CAR}(\mathbb{N}))_{X_{E}}$ with $\omega \leq \lambda \varphi$ for some $\lambda>0$. As before, there exists a projection $P \in \mathfrak{Z}_{\varphi}$ such that $\pi_{\omega}(\mathfrak{A})^{\prime \prime}=P \pi_{\varphi}(\mathfrak{A})^{\prime \prime}$, which implies that $\omega \in \mathcal{S}_{\mathbb{P}_{\mathbb{N}}}(\mathrm{CAR}(\mathbb{N}))_{X_{E}}$.

\section{SYMMETRIC STATES IN THE UNCOUNTABLE CASE}

The starting point to obtain the characterization of the extremal symmetric states is the clustering property given in Definition 5.1. It is the bridge between the extremality and the property to being a product state (cf. Theorem 5.3). Suppose that $\mathrm{CAR}(J)$ is generated by infinitely uncountably many annihilators, that is $J$ is uncountable. Fix a state $\varphi \in \mathcal{S}(\mathrm{CAR}(J))$. We generalize the clustering property in the following way.

We start with a pair $\mathcal{I}:=(I, \nu)$, where $I \subset J$ is countable and $\nu: I \rightarrow \mathbb{N}$ is a bijection defining an order $i_{1}, i_{2}, \ldots$ on $I$. Define the sequence $\left\{g_{n}^{\mathcal{I}}\right\}_{n \in \mathbb{N}} \subset \mathbb{P}_{J}$ as $g_{n}^{\mathcal{I}}:=g_{\nu\left(i_{n}\right)}$, where $g_{k} \in \mathbb{P}_{\mathbb{N}}$ is given in (5.1). Fix now $\varphi \in \mathcal{S}(\mathrm{CAR}(J))$. It is said strongly clustering if, for each $A, B \in \operatorname{CAR}(J)$ there exists $\mathcal{I}:=(I, \nu)$ with $A, B \in \mathrm{CAR}(I)$ such that

$$
\lim _{n} \varphi\left(\alpha_{g_{n}^{\mathcal{I}}}(A) B\right)=\varphi(A) \varphi(B) .
$$

As a preliminary fact we note that, if $\mathcal{I}:=(I, \nu)$ with $A \in \operatorname{CAR}(I)$ localized, and $g \in \mathbb{P}_{J}$, then there exists $n_{A, g}$ such that $n>n_{A, g}$ implies $\alpha_{g g_{n}^{\mathcal{I}}}(A)=\alpha_{g_{n}^{\mathcal{I}}}(A)$. In fact, as $g$ changes only a finite number of indices in $I$, say up to $m$, and $A$ is localized, say in the first $s$ elements of $I$, it is enough to choose $n$ such that $\max \{m, s\} \leq 2^{n-1}$. By using this fact, Lemma 5.2 holds true also in the present situation. Namely, fix $A \in \operatorname{CAR}(J)$. Then for each $\mathcal{I}=(I, \nu)$ such that $A \in \operatorname{CAR}(I)$

$$
\mathrm{w}-\lim _{n}\left[\pi_{\varphi}\left(\alpha_{g_{n}^{\mathcal{I}}}(A)\right) \Omega_{\varphi}\right]=\varphi(A) \Omega_{\varphi},
$$

provided $\varphi$ is extremal.

Now we are ready to extend Theorem 5.3 to uncountable case.

Theorem 6.1. Let $\varphi \in \mathcal{S}_{\mathbb{P}_{J}}(\operatorname{CAR}(J))$. Then $\varphi \in \mathcal{E}\left(\mathcal{S}_{\mathbb{P}_{J}}(\operatorname{CAR}(J))\right)$ if and only if $\varphi=\prod_{J} \rho$ for some even state $\rho \in \mathbb{M}_{2}(\mathbb{C})$.

Proof. If $\varphi$ is a product state, it is obviously strongly clustering. Suppose now $\varphi$ be strongly clustering and fix a sequence $\left\{A_{i}\right\}_{i \in \mathbb{N}} \subset \mathbb{M}_{2}(\mathbb{C})$. The proof proceeds by induction. Choose $\mathcal{I}:=(I, \nu)$ such that $A:=$ $\iota_{j_{1}}\left(A_{1}\right) \cdots \iota_{j_{n-1}}\left(A_{n-1}\right), B:=\iota_{j_{n}}\left(A_{n}\right)$ belong to $I$ and $(6.1)$ holds true. After noticing that

$$
\varphi\left(g_{n}^{\mathcal{I}}(A) B\right)=\varphi\left\lceil_{\mathrm{CAR}(I)}\left(g_{n}^{\mathcal{I}}(A) B\right) .\right.
$$


we can reduce the matter to the countable situation, that is to Theorem 5.3 , as $\operatorname{CAR}(I) \sim \operatorname{CAR}(\mathbb{N})$. Thus a symmetric state is a product one if and only if it is strongly clustering. On the other hand, if $\varphi$ is extremal we conclude by (6.2) that $\varphi$ is strongly clustering. For the reverse implication we reason as in Theorem 5.3 as well. Indeed, choose a vector $\xi \perp \Omega_{\varphi}$ belonging to $\mathcal{H}_{\varphi}^{\mathbb{P}_{J}}$ and fix $\varepsilon>0$. Then there exists $B \in$ $\operatorname{CAR}(J)$ such that $\left\|\xi-\pi_{\varphi}(B) \Omega\right\|<\varepsilon / 2$. Let $A \in \operatorname{CAR}(J)$ such that $\left\|\pi_{\varphi}(A)\right\| \leq 1$. We get for each $\mathcal{I}=(I, \nu)$ such that $A, B \in \operatorname{CAR}(I)$,

$$
\left|\left\langle\xi, \pi_{\varphi}(A) \Omega_{\varphi}\right\rangle\right| \leq\left|\varphi\left(\alpha_{g_{n}^{\mathcal{I}}}\left(A^{*}\right) B\right)\right|+\varepsilon / 2 .
$$

As $\varphi$ is strongly clustering, there exists an $\mathcal{I}:=(I, \nu)$ such that $A, B \in$ $\mathrm{CAR}(I)$ and (6.1) holds. Taking the limit on both sides in (6.3), one obtains

$$
\left|\left\langle\xi, \pi_{\varphi}(A) \Omega_{\varphi}\right\rangle\right| \leq \lim _{n}\left|\varphi\left(\alpha_{g_{n}^{\mathcal{I}}}\left(A^{*}\right) B\right)\right|+\varepsilon / 2=\left|\varphi\left(A^{*}\right)\right||\varphi(B)|+\varepsilon / 2<\varepsilon .
$$

We conclude as before that $\xi=0$ and then $\varphi$ is extremal. Since we verified that both properties (i.e. being a product state and extremeness) are equivalent to strong clustering, the proof is complete.

Remark 6.2. Theorem 6.1 holds true mutatis mutandis for the case of infinite tensor product of $C^{*}$-algebras considered in [31], when the index set is uncountable.

In the general (uncountable) case it is possible to achieve the ergodic decomposition of a symmetric state $\varphi$ as in Theorem 5.5. (countable case). In fact, the convex of the symmetric states on $\operatorname{CAR}(J)$ is yet a Choquet simplex (see [29], Theorem 3.1.14). The difference w.r.t. the countable case consists in the fact that $\mathcal{S}_{\mathbb{P}_{J}}(\mathrm{CAR}(J))$ is not metrizable. Then the unique maximal decomposing measure $\mu$ of $\varphi$ is only pseudosupported on $\mathcal{E}\left(\mathcal{S}_{\mathbb{P}_{J}}(\mathrm{CAR}(J))\right)$. Namely, we still have

$$
\varphi(A)=\int \psi(A) d \mu(\psi), \quad A \in \operatorname{CAR}(J),
$$

but here $\mathcal{E}\left(\mathcal{S}_{\mathbb{P}_{J}}(\mathrm{CAR}(J))\right.$ is merely a Borel set which is not a Baire one. Then the maximal measure $\mu$ satisfies $\mu(B)=1$ for each Baire set $B$ containing $\mathcal{E}\left(\mathcal{S}_{\mathbb{P}_{J}}(\mathrm{CAR}(J))\right.$ (see [8], Theorem 4.1.11).

Finally, one realizes that most of the results at the end of Section 5, except Remark 5.9, may be extended to the general (uncountable) case. For example, as in Proposition 5.6, one sees that the simplex of the symmetric states on $\mathrm{CAR}(J)$ has a *-weakly closed boundary and is affinely isomorphic to the regular probability measures on the unit interval. 


\section{ACKNOWLEDGEMENTS}

The second-named author would like to thank Simion Stoilow Institute of Mathematics of the Romanian Academy for the warm hospital-

ity, thanks to a BITDEFENDER invited professorship position, where part of the present work has been done.

\section{REFERENCES}

[1] Accardi L., Ben Ghorbal A., Crismale V., Lu Y. G. Singleton conditions and quantum De Finetti's theorem, Infin. Dimens. Anal. Quantum Probab. Relat. Top. 11 (2008), 639-660.

[2] Accardi L., Fidaleo F., Mukhamedov F. Quantum Markov states and chains on the CAR algebras, Infin. Dimens. Anal. Quantum Probab. Relat. Top. 10 (2007), 165-183.

[3] Accardi L., Lu Y.G. A continuous version of De Finetti's theorem, Ann. Probab. 21 (1993), 1478-1493.

[4] Araki H., Moriya H. Equilibrium statistical mechanics of Fermion lattice systems, Rev. Math. Phys. 15 (2003), 93-198.

[5] Araki H., Moriya H. Joint extension of states of subsystems for a CAR system, Commun. Math. Phys. 237 (2003), 105-122.

[6] Barreto S. D., Fidaleo F. Disordered Fermions on lattices and their spectral properties, J. Stat. Phys. 143 (2011), 657-684.

[7] Bauer H. Schilowscher rand und dirichletsches problem, Ann. Inst. Fourier 11 (1961), 89-136.

[8] Bratteli O., Robinson D. W. Operator algebras and quantum statistical mechanics I, Springer, Berlin-Heidelberg-New York, 1981.

[9] Bratteli O., Robinson D. W. Operator algebras and quantum statistical mechanics II, Springer, Berlin-Heidelberg-New york, 1981.

[10] Connes A. Une classification des facteurs de type III, Ann. Scient. Éc. Norm. Sup. 6 (1973), 133-252.

[11] Christandl M., König R., Mitchison G., Renner R. One-and-a-half quantum de Finetti theorems, Comm. Math. Phys. 273 (2007), 473-498.

[12] Christandl M., Toner B. Finite de Finetti theorem for conditional probability distributions describing physical theories, J. Math. Phys. 50 (2009), no. 4, 042104, 11 pp.

[13] De Finetti B. Funzione caratteristica di un fenomeno aleatorio, Atti Accad. Naz. Lincei, VI Ser., Mem. Cl. Sci. Fis. Mat. Nat. 4 (1931), 251-259.

[14] Diaconis P., Freedman D. A. Finite exchangeable sequences, Ann. Prob. 8 (1980), 745-764.

[15] Fannes M., Lewis J. T., Verbeure A. Symmetric stats of composite systems, Lett. Math. Phys. 15 (1988), 255-260.

[16] Fidaleo F. Fermi Markov states, J. Operator Theory, 66 (2011), 385-414.

[17] Freedman D. A. Invariance under mixing which generalize De Finetti's theorem: Continuous time parameter, Ann. Math. Stat. 34 (1963), 1194-1216.

[18] Hewitt E., Savage L. F. Symmetric measures on Cartesian products, Trans. Amer. Math. Soc. 80 (1955), 470-501. 
[19] Hudson R. L., Moody G. R. Locally normal symmetric states and an analogue of De Finetti's theorem, Z. Wahr. Verw. Gebiete 33 (1976), 343-351.

[20] König R., Mitchison G. A most compendious and facile quantum de Finetti theorem, J. Math. Phys. 50 (2009), no. 1, 012105, 20 pp.

[21] Köstler C. A noncommutative extended De Finetti theorem, J. Funct. Anal. 258 (2010), 1073-120.

[22] Köstler C, Speicher R. A noncommutative De Finetti theorem: invariance under quantum permutations is equivalent to freeness with amalgamation, Commun. Math. Phys. 291 (2009), 473-490.

[23] Lehner F. Cumulants in noncommutative probability theory IV. noncrossing cumulants: De Finetti's theorem and $L^{p}$-inequalities, J. Funct. Anal. 239 (2006), 214-246.

[24] Leverrier A., Cherf N. J. A quantum De Finetti theorem in phase space representation, Phys. Rev. A 80010102 (2009).

[25] Leverrier A., Karpov E., Grangier P., Cherf N. J. Security of continuousvariable QKD: exploiting symmetries in phase space, New Journal of Physics 11115009 (2009)

[26] Matsui T. Ground states of Fermions on lattices, Commun. Math. Phys. 182 (1996), 723-751.

[27] Nielsen O. A. Direct integral theory, Marcel Dekker, New York-Basel, 1980.

[28] Renner R., Cirac I. J. A de Finetti representation theorem for infinite dimensional quantum systems and applications to quantum cryptography, Phys. Rev. Lett. 102110504 (2009).

[29] Sakai S. $C^{*}$-algebras and $W^{*}$-algebras, Springer, Berlin-Heidelberg-New York 1971.

[30] Størmer E. Large groups of automorphisms of $C^{*}$-algebras, Commun. Math. Phys. 5 (1967), 1-22.

[31] Størmer E. Symmetric states of infinite tensor products of $C^{*}$-algebras, J. Funct. Anal. 3 (1969), 48-68.

[32] Strătilă S. Modular theory in operator algebras, Abacus press, Tunbridge Wells, Kent (1981).

[33] Strătilă S., Zsidó, L. Lectures on von Neumann algebras, Abacus press, Tunbridge Wells, Kent, (1979).

[34] Sutherland C. Crossed products, direct integrals and Connes' classification of type III factors, Math. Scand. 40 (1977), 209-214.

[35] Takesaki M. Theory of operator algebras I, Springer, Berlin-Heidelberg-New York 1979.

[36] Takesaki M. Theory of operator algebras III, Springer, Berlin-HeidelbergNew York 1979.

Vitonofrio Crismale, Dipartimento di Matematica, Università degli STUdi DI BARI, Via E. ORABONA, 4, 70125 BARI, ITALY

E-mail address: crismalev@dm.uniba.it

Francesco Fidaleo, Dipartimento di Matematica, Università di Roma Tor Vergata, Via della Ricerca Scientifica 1, Roma 00133, Italy

E-mail address: fidaleo@mat.uniroma2.it 